\title{
Phellodendron and Citrus extracts benefit cardiovascular health in osteoarthritis patients: a double-blind, placebo-controlled pilot
} study

\author{
Julius Oben ${ }^{1}$, Ebangha Enonchong1, Shil Kothari², Walter Chambliss ${ }^{3}$, \\ Robert Garrison ${ }^{4}$ and Deanne Dolnick*4
}

\begin{abstract}
Address: ${ }^{1}$ Laboratory of Nutrition \& Nutritional Biochemistry, Department of Biochemistry, University of Yaounde I, Cameroon, ${ }^{2}$ Gateway Health Alliances, Inc, 4769 Mangels Blvd, Fairfield, CA, 94534, USA, ${ }^{3}$ University of Mississippi, University, MS, 38677, USA and ${ }^{4}$ Next Pharmaceuticals, 360 Espinosa Road, Salinas, CA, 93907, USA
\end{abstract}

Email: Julius Oben - juliusoben@hotmail.com; Ebangha Enonchong - juliusoben@hotmail.com; Shil Kothari - shilkothari@hotmail.com; Walter Chambliss - chamblissw@aol.com; Robert Garrison - ddolnick@nextpharmaceuticals.com;

Deanne Dolnick* - ddolnick@nextpharmaceuticals.com

* Corresponding author

Published: 20 May 2008

Nutrition Journal 2008, 7:16 doi:10.1186/1475-2891-7-16
Received: 3 October 2007

Accepted: 20 May 2008

This article is available from: http://www.nutritionj.com/content/7/1/16

(c) 2008 Oben et al; licensee BioMed Central Ltd.

This is an Open Access article distributed under the terms of the Creative Commons Attribution License (http://creativecommons.org/licenses/by/2.0), which permits unrestricted use, distribution, and reproduction in any medium, provided the original work is properly cited.

\begin{abstract}
Background: The objective of this clinical study was to assess the potential benefit of a dietary supplement, NP 06-I, on cardiovascular protective properties in overweight and normal weight adults diagnosed with osteoarthritis of the knee.

Methods: An 8-week, placebo-controlled, randomized, double-blind study was conducted with four groups, comparing the effects of NP 06-I to placebo in overweight and normal weight subjects diagnosed with primary osteoarthritis of the knee. NP 06-I (a combination of two botanical extracts; Phellodendron amurense bark and Citrus sinensis peel) or matching placebo was given in a dose of two capsules ( $370 \mathrm{mg}$ each) twice daily. The outcome measures reported are lipid levels, weight, BMI, blood pressure and fasting glucose. Analyses of variance were used to compare changes of physiological measures over the trial period and between groups.

Results: Eighty (80) subjects were enrolled and 45 subjects completed the study. No serious adverse events were reported. NP 06-I administration was associated with a general improvement in lipid levels. Both the overweight and normal weight treatment groups had significant reductions in triglycerides and LDL-cholesterol, as well as a significant increase in HDL-cholesterol compared to their respective control groups.
\end{abstract}

Overall there were decreases in blood pressure in both overweight and normal weight treatment groups compared to respective placebo groups. There was also a significant decrease in fasting glucose levels in the overweight treatment group compared to the start of the study and to the overweight placebo group. There was no change in fasting blood sugar for the normal weight groups.

Both overweight and normal weight treatment groups lost a significant amount of weight compared to their respective placebo groups. The overweight treatment group lost an average of $5 \%$ body weight after 8 weeks, which was associated with a significant loss in BMI over time.

Conclusion: In this pilot study NP 06-I had a beneficial effect on cardiovascular risk factors; namely lipid levels, blood pressure and fasting glucose levels. Administration of NP 06-I was also associated with weight loss. 


\section{Background}

Osteoarthritis, the most common form of arthritis, is characterized by degradation of articular cartilage which manifests as joint pain followed by reduced mobility. The origin of the disease is unknown but obesity, joint injury, metabolic diseases, bone and joint malfunctions, genetic factors and age have been implicated. Therapies for osteoarthritis include weight control, physiotherapy and pharmacological agents. Conventional drug treatments include analgesics, anti-inflammatory agents, disease modifying therapies, hyaluronic acid, intra-articular glucocorticoids and topical analgesic/anti-inflammatory agents [1]. More recently there has been a focus on nutritional support. Recent systematic reviews highlight the scientific evidence for potential nutritional and herbal preparations for those with osteoarthritis [2,3].

A systematic review of the literature covering obese subjects diagnosed with osteoarthritis of the knee concluded that osteoarthritis related disability could be significantly improved with a loss of over $5.1 \%$ body weight [4]. Besides osteoarthritis related disability, increased body weight is associated with higher incidence of diabetes, hypertension and cardiovascular disease. This array of potential disease risks associated with excess weight has been termed metabolic syndrome. Individuals with three or more of the components of central obesity (excess fat mainly in the abdominal area), hyperinsulinemia, dyslipidemia and hypertension are considered to have metabolic syndrome. Treatment involves lifestyle changes such as diet and exercise, as well as intervention with prescription drugs [5].

The subject of this study, NP06-1 (Flavoxine (TM $^{\mathrm{TM}}$ Citrofen $^{\mathrm{TM}}$, Next Pharmaceuticals, Inc., Salinas, CA), is a proprietary product consisting of a blend of extracts of Phellodendron amurense tree bark and Citrus sinensis (orange) peel standardized to berberine and polymethoxylated flavones (PMFs), respectively. NP06-1 was formulated with the goal of combining the beneficial effects of both the phellodendron and orange peel extracts. There are indications in the literature, as well as preliminary unpublished studies conducted by Next Pharmaceuticals, that this combination might reduce inflammation as well as have beneficial effects on lipid levels.

The objective of this clinical study was to study the effects of NP 06-1 compared to placebo on biomarkers related to cardiovascular health. Both overweight and normal weight subjects were studied to determine whether or not there might be a difference in benefits to these two groups. The effects of NP06-1 compared to placebo in the management of joint pain and mobility caused by osteoarthritis of the knee will be the subject of another publication.

\section{Methods \\ Study Design}

The clinical trial was designed as a placebo-controlled, randomized, double-blind study with four groups. Forty overweight and forty normal weight subjects were enrolled into either treatment or placebo groups, with twenty subjects in each group as shown in Table 1. Subjects were recruited via advertisements at the University of Yaounde I Teaching Hospital, the Djongolo Baptist Hospital and through the public media. The IRB of the Faculty of Medicine and Biomedical Sciences of the University of Yaounde I in Cameroon approved the study and all subjects provided informed consent. Dr. Julius Oben, Associate Professor of Nutritional Biochemistry at the University of Yaounde I, Cameroon was the principal investigator.

To be included in the study, participants needed to be men or women, 25 to 60 years of age, diagnosed with primary osteoarthritis of the target knee using the American College of Rheumatology guidelines [6] by the treating physician and confirmed by the clinical investigator, and with a BMI between $25 \mathrm{~kg} / \mathrm{m}^{2}$ to $40 \mathrm{~kg} / \mathrm{m}^{2}$ (Overweight Groups) or $18.9 \mathrm{~kg} / \mathrm{m}^{2}$ to $24.9 \mathrm{~kg} / \mathrm{m}^{2}$ (Normal Weight Groups).

The exclusion criteria were: morbid obesity BMI $>40 \mathrm{~kg} /$ $\mathrm{m}^{2}$, diagnosed with rheumatoid arthritis, joint replacements in any one of the knees, unable to walk without assistance, enrollment in another clinical study in the past 6 months, pregnancy, active infection, autoimmune disease, AIDS, HIV, active hepatitis, active malignancy, or diabetes requiring daily insulin management.

\section{Administration and Dosage}

NP 06-1 is a proprietary product of Next Pharmaceuticals, Salinas, California. It is a blend of Phellodendron amurense Rupr. [Rutaceae] tree bark extract standardized to a minimum of $50 \%$ berberine and Citrus sinensis (L.) Osbeck [Rutaceae] peel extract standardized to a minimum of $30 \%$ polymethoxylated flavones (PMF). Berberine and PMFs were chosen as representative chemical constituents because they have demonstrated biological activity. Next Pharmaceuticals has licensed US Patent Nos. 6,184,246

Table I: Study Groups.

\begin{tabular}{clcc}
\hline Group & BMI Category & Treatment & Subjects \\
\hline OP & Overweight* & Placebo & $20(13)$ \\
OT & Overweight* & Active & $20(14)$ \\
NP & Normal weight $\dagger$ & Placebo & $20(7)$ \\
NT & Normal weight $\dagger$ & Active & $20(11)$ \\
\hline
\end{tabular}

*BMI $25 \mathrm{~kg} / \mathrm{m}^{2}-40 \mathrm{~kg} / \mathrm{m}^{2}$

† BMI $18.9 \mathrm{~kg} / \mathrm{m}^{2}-24.9 \mathrm{~kg} / \mathrm{m}^{2}$

The number of subjects initially enrolled is listed; with the number that completed the study in parenthesis. 
and $6,987,125$ obtained by the USDA for actions described by PMFs. NP 06-1 is sold under the trade names Flavoxine $^{\mathrm{TM}}$ and Citrofen ${ }^{\mathrm{TM}}$.

Subjects were allocated into groups using a random number table and instructed to take two NP 06-1 capsules (370 mg formula per capsule) or matching placebo (identical red two-piece hard shell capsules) with food in the morning and at night ( 4 capsules per day) for a total of 8 weeks.

Subjects were instructed to avoid taking analgesics (a 5 day wash-out period prior to enrollment) or cholesterol lowering medications (a 30 day wash-out period prior to enrollment) during the study and to stay with their normal exercise and diet regimens.

\section{Study Variables}

The study variables were biomarkers of cardiovascular health: weight, BMI, triglycerides, total cholesterol, HDLcholesterol, LDL-cholesterol, systolic/diastolic blood pressure and fasting glucose.

\section{Data Collection}

Blood samples $(8 \mathrm{ml})$ were collected by venous puncture at time 0,4 and 8 weeks. Subjects were requested to come to the study center on the mornings of each site visit after a 12-hour fast. The concentrations of triglycerides, HDLcholesterol, LDL-cholesterol and fasting glucose, were measured using commercial diagnostic kits (triglyceride Infinity, EZ HDL ${ }^{\mathrm{TM}}$ cholesterol, EZ LDL ${ }^{\mathrm{TM}}$ cholesterol, Glucose Trinder) from SIGMA Diagnostics. Body weight was determined in 12-hour fasted subjects using a Tanita ${ }^{\mathrm{TM}}$ scale and height was measured using a stadiometer.

\section{Safety Assessment}

Subjects were given three emergency telephone numbers to contact during the conduct of the study, if they had any adverse events or other concerns related to the study. Each subject was interviewed during site visits to solicit information on possible adverse effects they might have encountered. Participants were instructed to inform the investigator, if the reason for dropping out of the study was due to adverse effects.

\section{Statistical Analysis}

An analysis of variance model, using repeated measures, was used to compare changes of physiological measures over the trial period and between groups. All statistics were 2 -tailed and significance was set at $\mathrm{p}<0.05$.

\section{Results}

Eighty (80) subjects were enrolled in the study and randomized into four groups designated OP (Overweight Placebo), OT (Overweight Treatment), NP (Normal weight Placebo) and NT (Normal weight Treatment) (Table 1). Forty five (45) subjects completed the study and the reasons given by the dropouts are given in Table 2. The dropouts were fairly evenly distributed among the groups, with the exception of Group NP which lost nearly twice as many participants as the other groups. The majority of Group NP dropouts cited no improvement in their condition as their reason for dropping out. There is no indication that their demographics were different from those

Table 2: Reasons given by subjects for dropping out of the study

\begin{tabular}{|c|c|c|}
\hline Group & No. Subjects & Reason for drop out \\
\hline \multirow[t]{4}{*}{ OP } & 2 & Reported no improvement in their condition \\
\hline & I & Nausea and vomiting; Malaria attack \\
\hline & I & Moved out of town \\
\hline & 3 & No reason given \\
\hline \multirow[t]{4}{*}{ OT } & 1 & Reported improvement as too slow \\
\hline & I & Tested positive for hepatitis \\
\hline & I & Moved out of town \\
\hline & 3 & No reason given \\
\hline \multirow[t]{5}{*}{ NP } & 7 & Reported no improvement in their condition \\
\hline & I & Nausea \\
\hline & I & Started weight management program \\
\hline & 1 & Started fasting and stopped treatment \\
\hline & 3 & No reason given \\
\hline \multirow[t]{4}{*}{$\mathbf{N T}$} & 1 & Malaria attack \\
\hline & I & Nausea \\
\hline & 1 & Started fasting and stopped treatment \\
\hline & 6 & No reason given \\
\hline
\end{tabular}


who stayed in the study. No serious adverse events were reported by any of the dropouts or by those who completed the study.

Baseline lipid levels for the overweight and normal weight groups were in the normal range. The average levels for total cholesterol were 194.5 to $197.3 \mathrm{mg} / \mathrm{dl}$, HDL-cholesterol 63.6 to $69.8 \mathrm{mg} / \mathrm{dl}$, LDL-cholesterol 89.3 to 123.6 $\mathrm{mg} / \mathrm{dl}$ and triglycerides 86.0 to $124.8 \mathrm{mg} / \mathrm{dl}$. Baseline blood pressure levels for the overweight and normal weight groups were in the upper limits of the normal range, with average levels for systolic blood pressure from 126.6 to $135.8 \mathrm{mmHg}$ and those for diastolic blood pressure from 81.2 to $84.7 \mathrm{mmHg}$. Baseline fasting blood sugar values for the groups averaged 56.9 to $113.8 \mathrm{mg} / \mathrm{dl}$, also in the normal range.

\section{Weight/BMI}

In Group OT, the subjects lost a significant amount of weight and their BMI was reduced compared to the start of the study ( $\mathrm{p}<0.001$ at 4 and 8 weeks) (Table 3 ). They lost an average of $2.5 \mathrm{~kg}$ (5.5 pounds, $3.1 \%$ of body weight) after 4 weeks and an average of $4.2 \mathrm{~kg}(9.2$ pounds, $5.1 \%$ of body weight) after 8 weeks. There were smaller, but significant, changes in weight and BMI from baseline for Group OP. Comparisons of weight loss between overweight groups revealed that the treatment group lost on average 3.7 times as much weight as the placebo group at 8 weeks ( 9.2 pounds versus 2.5 pounds) (p $<0.001$ ). However, in comparison, the changes in BMI's between the two overweight groups were not significant (Table 4).

In Group NT there was a significant decrease in weight and BMI at 8 weeks $(\mathrm{p}<0.05)$ compared to baseline
(Table 3). At 8 weeks, this group lost an average of $1.18 \mathrm{~kg}$ ( 2.6 pounds, $1.8 \%$ of body weight). Group NP also had a significant loss of weight and BMI after 8 weeks compared to the start of the study $(p<0.01)$. Comparisons of weight loss between normal weight groups revealed that the treatment group lost on average 1.5 times as much weight as the placebo group at 8 weeks (2.6 pounds versus 1.7 pounds) ( $\mathrm{p}<0.01)$. There was no significant difference in change in BMI's between the normal weight treatment and placebo groups (Table 4).

\section{Total Cholesterol}

For Group OT, there were significant decreases in total serum cholesterol levels at 4 weeks $(15.6 \%)$ and 8 weeks $(21.6 \%)$ compared to baseline (both p < 0.001) (Table 5). For Group OP, there were smaller, but significant decreases in total serum cholesterol levels at 4 weeks $(7.2 \%)$ and 8 weeks $(8.0 \%)$ compared to baseline ( $\mathrm{p}<$ 0.01 and $\mathrm{p}<0.05$, respectively). Subjects in the treatment group had a 2.7 times greater average decrease in total cholesterol in comparison to the placebo group. However the individual data was highly variable and the change in Group OT compared to that in Group OP was not significant (Table 4).

For Group NT there were significant decreases in total serum cholesterol at 4 weeks $(7.3 \%)$ and 8 weeks $(9.7 \%)$ compared to baseline (both $\mathrm{p}<0.01$ ). For Group NP there was a significant decrease at 8 weeks $(7.3 \%)$ but no significant change after 4 weeks (Table 5). There were no significant differences between the normal weight groups.

\section{LDL-Cholesterol}

For Group OT, there were significant decreases in LDLcholesterol levels at 4 weeks (31.0\%) and 8 weeks

Table 3: Body Weight, BMI.

\begin{tabular}{|c|c|c|c|c|c|}
\hline Group & Time 0 & 4 weeks & 8 weeks & $\% \Delta 0-4$ wks & $\% \Delta 0-8$ wks \\
\hline \multicolumn{6}{|l|}{ OP } \\
\hline Weight (kg) & 85.0 & $-1.14 \pm 0.96$ & $-1.15 \pm 1.30$ & $-1.3^{*}$ & $-1.4^{*}$ \\
\hline BMI $\left(\mathrm{kg} / \mathrm{m}^{2}\right)$ & 31.1 & $-0.42 \pm 0.59$ & $-0.42 \pm 0.32$ & $-1.3^{*}$ & $-1.4^{*}$ \\
\hline \multicolumn{6}{|l|}{ OT } \\
\hline Weight (kg) & 81.7 & $-2.49 \pm 1.09$ & $-4.19 \pm 1.24$ & $-3.1^{*}$ & $-5.1^{*}$ \\
\hline BMI $\left(\mathrm{kg} / \mathrm{m}^{2}\right)$ & 31.7 & $-0.94 \pm 0.11$ & $-1.59 \pm 0.11$ & $-3.0^{*}$ & $-5.1^{*}$ \\
\hline \multicolumn{6}{|l|}{ NP } \\
\hline Weight (kg) & 67.1 & $-0.27 \pm 0.60$ & $-0.76 \pm 0.34$ & -0.4 & $-1.1^{*}$ \\
\hline BMI $\left(\mathrm{kg} / \mathrm{m}^{2}\right)$ & 24.0 & $-0.10 \pm 0.05$ & $-0.28 \pm 0.04$ & -0.4 & $-1.2^{*}$ \\
\hline \multicolumn{6}{|l|}{ NT } \\
\hline Weight (kg) & 67.0 & $-0.51 \pm 1.08$ & $-1.18 \pm 1.29$ & -0.8 & $-1.8^{*}$ \\
\hline BMI $\left(\mathrm{kg} / \mathrm{m}^{2}\right)$ & 24.8 & $-0.18 \pm 0.05$ & $-0.42 \pm 0.08$ & -0.7 & $-1.7^{*}$ \\
\hline
\end{tabular}

*Significant $P$ values $<0.05$.

$\mathrm{OP}=$ overweight placebo, $\mathrm{OT}=$ overweight treatment, $\mathrm{NP}=$ normal weight placebo and NT = normal weight treatment.

Values at the start of the study (Time 0) listed in the first column are means of data from individuals. Mean changes after 4 weeks and 8 weeks are listed in the second and third columns as \pm Standard Deviation. A negative value indicated a decrease in value and a positive number represents an increase in value. $\% \Delta$ values are percent mean change in value between time 0 and either 4 or 8 weeks. Values* are significant $\mathrm{P}$-value comparisons $(<0.05)$ between initial values at Time 0 and either 4 weeks or 8 weeks. 
Table 4: Inter-Group Analysis Comparing the Paired Treatment to Placebo Groups.

\begin{tabular}{|c|c|c|c|c|}
\hline & \multicolumn{2}{|c|}{ Group OP vs. Group 0T } & \multicolumn{2}{|c|}{ Group NT vs. Group NP } \\
\hline & $\mathrm{T}=4$ weeks & $\mathbf{T}=8$ weeks & $\mathrm{T}=4$ weeks & $\mathbf{T}=\mathbf{8}$ weeks \\
\hline Weight & $p<0.01$ & $p<0.001$ & $p<0.05$ & $p<0.01$ \\
\hline $\mathrm{BMI}$ & NS & NS & NS & NS \\
\hline Cholesterol & $\mathrm{P}<0.001$ & NS & NS & NS \\
\hline HDL & NS & $p<0.001$ & $p<0.05$ & $P<0.05$ \\
\hline LDL & NS & $p<0.01$ & NS & $p<0.01$ \\
\hline Triglycerides & NS & $\mathrm{p}<0.001$ & $P<0.01$ & $\mathrm{p}<0.01$ \\
\hline Systolic BP & NS & $p<0.05$ & $p<0.05$ & NS \\
\hline Diastolic BP & $p<0.05$ & $p<0.001$ & $p<0.05$ & $p<0.001$ \\
\hline Glucose & $p<0.001$ & $p<0.001$ & NS & NS \\
\hline
\end{tabular}

P-value comparisons are between overweight placebo (OP) and overweight treatment (OT) groups and between normal-weight placebo groups (NP) and normal-weight treatment (NT) groups at 4 and 8 weeks. NS $=$ not significant.

(44.6\%) compared to baseline (both $\mathrm{p}<0.001)$. For Group OP, there were smaller, but significant decreases at 4 weeks $(13.8 \%)$ and 8 weeks $(14.2 \%)$ compared to baseline (both $\mathrm{p}<0.05)$ (Table 5). Subjects in the treatment group had an average of 3.1 times greater decrease in LDL in comparison to the placebo group at 8 weeks $(\mathrm{p}<0.01)$.

For Group NT there were significant decreases in LDL-cholesterol levels at 4 weeks $(11.0 \%)$ and 8 weeks $(16.8 \%)$ compared to baseline ( $\mathrm{p}<0.05$ and 0.01 , respectively). For subjects in Group NP there was a significant decrease in LDL at 8 weeks $(14.5 \%$; $<0.05)$; with no significant change after 4 weeks (Table 5). Comparison of normal weight groups showed a significant difference between treatment and placebo at 8 weeks $(\mathrm{p}<0.01)$.

\section{HDL-Cholesterol}

For Group OT, there was a significant increase in HDLcholesterol levels at 8 weeks $(11.8 \%)$ compared to baseline $(\mathrm{p}<0.05)$. There was no significant change at 4 weeks. For Group OP, there were no significant changes in HDL (Table 5). Subjects in the treatment group had an

Table 5: Lipid Levels.

\begin{tabular}{|c|c|c|c|c|c|}
\hline Group & Time 0 & 4 weeks & 8 weeks & $\% \Delta 0-4$ wks & $\% \Delta 0-8$ wks \\
\hline \multicolumn{6}{|l|}{ OP } \\
\hline Cholesterol & 195.7 & $-13.30 \pm 12.20$ & $-14.80 \pm 16.83$ & $-7.2^{*}$ & $-8.0^{*}$ \\
\hline $\mathrm{HDL}$ & 69.8 & $0.86 \pm 0.95$ & $-0.87 \pm 5.72$ & 1.2 & -1.3 \\
\hline LDL & 89.3 & $-12.64 \pm 16.49$ & $-13.01 \pm 16.89$ & $-13.8^{*}$ & $-14.2^{*}$ \\
\hline Triglycerides & 124.8 & $-7.85 \pm 10.5$ & $-4.58 \pm 7.82$ & $-6.3^{*}$ & $-3.7^{*}$ \\
\hline \multicolumn{6}{|l|}{ OT } \\
\hline Cholesterol & 194.5 & $-31.6 \pm 20.68$ & $-43.45 \pm 20.96$ & $-15.8 *$ & $-21.7^{*}$ \\
\hline $\mathrm{HDL}$ & 69.7 & $3.37 \pm 10.64$ & $8.33 \pm 13.25$ & 4.8 & $11.8^{*}$ \\
\hline LDL & 105.9 & $-32.99 \pm 23.61$ & $-47.47 \pm 22.24$ & $-31.0 *$ & $-44.6 *$ \\
\hline Triglycerides & 118.4 & $-16.52 \pm 21.78$ & $-21.57 \pm 22.97$ & $-13.9 *$ & $-18 .\left.\right|^{*}$ \\
\hline \multicolumn{6}{|l|}{ NP } \\
\hline Cholesterol & 197.3 & $-9.83 \pm|2.9|$ & $14.88 \pm 13.37$ & -4.8 & $-7.3^{*}$ \\
\hline $\mathrm{HDL}$ & 63.6 & $-0.36 \pm 6.91$ & $4.3 \pm 4.88$ & -0.6 & 6.8 \\
\hline LDL & 123.6 & $-8.82 \pm 17.36$ & $-18.02 \pm 14.38$ & -7.1 & $-14.5^{*}$ \\
\hline Triglycerides & 86.0 & $-3.27 \pm 2.38$ & $-5.83 \pm 8.30$ & $-3.8^{*}$ & -6.8 \\
\hline \multicolumn{6}{|l|}{ NT } \\
\hline Cholesterol & 197.0 & $-|4.77 \pm| 5.5 \mid$ & $-19.45 \pm 16.26$ & $-7.3^{*}$ & $-9.7^{*}$ \\
\hline $\mathrm{HDL}$ & 67.3 & $0.50 \pm 4.58$ & $2.63 \pm 2.96$ & 0.74 & $3.9^{*}$ \\
\hline LDL & 116.0 & $-12.80 \pm 15.86$ & $-19.51 \pm 16.89$ & $-11.0 *$ & $-16.8^{*}$ \\
\hline Triglycerides & 88.7 & $-12.33 \pm 6.50$ & $-12.82 \pm 6.03$ & $-13.9 *$ & $-14.5^{*}$ \\
\hline
\end{tabular}

*Significant $P$ values $<0.05$.

$\mathrm{OP}=$ overweight placebo, $\mathrm{OT}=$ overweight treatment, $\mathrm{NP}=$ normal weight placebo and $\mathrm{NT}=$ normal weight treatment.

Values at the start of the study (Time 0 ) listed in the first column are means of data from individuals. Mean changes after 4 weeks and 8 weeks are listed in the second and third columns as \pm Standard Deviation. A negative value indicated a decrease in value and a positive number represents an increase in value. $\% \Delta$ values are percent mean change in value between time 0 and either 4 or 8 weeks. Values* are significant P-value comparisons $(<0.05)$ between initial values at Time 0 and either 4 weeks or 8 weeks. 
average of 9 times greater increase in comparison to the placebo group at 8 weeks $(\mathrm{p}<0.001)$.

For Group NT there was a significant increase in HDL-cholesterol levels at 8 weeks (3.9\%) compared to baseline (p $<0.05$ ). There were no significant changes in HDL levels compared to the start of the study in Group NP. Comparison of normal weight groups showed a significant difference between treatment and placebo at 4 and 8 weeks (both $\mathrm{p}<0.05)$ (Table 4).

\section{Triglycerides}

For Group OT, there was a significant decrease in plasma triglyceride levels at 4 weeks $(13.9 \%)$ and 8 weeks $(18.1 \%)$ compared to baseline (both $\mathrm{p}<0.05)$. Group OP also had a significant decrease in triglyceride levels at 4 weeks and 8 weeks compared to baseline (both $\mathrm{p}<0.05$ ) (Table 5). However there was a greater decrease in the treatment group in comparison to the placebo group at 8 weeks $(\mathrm{p}<0.001)$.

For Group NT, there was a significant decrease in triglyceride levels at 4 weeks $(13.9 \%)$ and 8 weeks $(14.5 \%)$ compared to baseline (both $\mathrm{p}<0.001$ ). For Group NP, there was a significant decrease in triglycerides at 4 weeks but not at 8 weeks. Comparison of normal weight groups showed a significant difference between treatment and placebo at 4 and 8 weeks (both $\mathrm{p}<0.01$ ).

\section{Blood Pressure}

For Group OT, there was a significant decrease in systolic blood pressure at 4 weeks $(3.3 \%)$ and 8 weeks $(6.0 \%)$ compared to baseline (both $\mathrm{p}<0.05$ ). There was also a significant decrease in diastolic blood pressure at 4 weeks $(8.3 \%)$ and 8 weeks $(13.1 \%)$ compared to baseline $(\mathrm{p}<$ 0.01 and $\mathrm{p}<0.001$, respectively). There were no significant changes in either systolic or diastolic blood pressure in Group OP (Table 6). Comparison of overweight groups showed a significant difference between treatment and placebo in diastolic blood pressure at both 4 and 8 weeks ( $\mathrm{p}<0.05$ and $\mathrm{p}<0.001$, respectively) and in systolic blood pressure at 8 weeks $(\mathrm{p}<0.05)($ Table 4$)$.

For Group NT, there were no significant changes in systolic blood pressure compared to the beginning of the study. However there were significant decreases in diastolic blood pressure at 4 weeks $(9.1 \%)$ and 8 weeks $(11.6 \%)$ compared to baseline ( $\mathrm{p}<0.05$ and $\mathrm{p}<0.01$, respectively). For Group NP, there was a significant decrease in systolic blood pressure at 8 weeks $(p<0.05)$ but not at 4 weeks. This group had significant decreases in diastolic blood pressure at both 4 and 8 weeks $(\mathrm{p}<0.001$ and 0.01 , respectively). Comparison of normal weight groups showed a significant difference between treatment and placebo in diastolic blood pressure at both 4 and 8 weeks ( $\mathrm{p}<0.05$ and $\mathrm{p}<0.001$, respectively). Comparison of Groups NT to NP also showed a significant difference in systolic blood pressure at 4 weeks $(\mathrm{p}<0.05)$, but not at 8 weeks (Table 4).

Table 6: Blood Pressure, Fasting Glucose.

\begin{tabular}{lccccc}
\hline $\begin{array}{c}\text { Group } \\
\text { OP }\end{array}$ & Time 0 & 4 weeks & 8 weeks & $\% \Delta 0-4$ wks & $\% \Delta 0-8$ wks \\
Systolic BP & 130.8 & $1.31 \pm 12.07$ & $2.15 \pm 8.66$ & 1.0 & 1.6 \\
$\begin{array}{l}\text { Diastolic BP } \\
\text { Glucose }\end{array}$ & 81.8 & $-3.46 \pm 7.47$ & $-2.31 \pm 8.62$ & -4.2 & -2.8 \\
OT & 113.8 & $0.14 \pm 7.77$ & $-9.64 \pm 8.99$ & 0.2 & -10.7 \\
Systolic BP & 135.1 & $-4.43 \pm 8.66$ & $-8.07 \pm 11.13$ & $-3.3^{*}$ & $-6.0^{*}$ \\
Diastolic BP & 82.7 & $-6.86 \pm 6.98$ & $-10.85 \pm 9.00$ & $-8.3^{*}$ & $-13.1^{*}$ \\
Glucose & 73.4 & $-8.58 \pm 11.28$ & $-17.67 \pm 11.69$ & $-9.5^{*}$ & $-19.6^{*}$ \\
Systolic BP & 127.0 & $-5.66 \pm 9.56$ & $-6.66 \pm 8.08$ & -4.5 & $-5.3^{*}$ \\
Diastolic BP & 84.7 & $-7.55 \pm 2.07$ & $-10.50 \pm 5.40$ & $-8.85^{*}$ & $-12.39 *$ \\
Glucose & 57.6 & $-0.18 \pm 5.6$ & $-0.02 \pm 3.98$ & -0.3 & -0.0 \\
NT & & & & -1.8 \\
Systolic BP & 126.6 & $2.90 \pm 12.60$ & $-2.30 \pm 9.21$ & -9.3 & $-1.6^{*}$ \\
Diastolic BP & 81.2 & $-7.36 \pm 9.34$ & $-9.45 \pm 9.96$ & -0.9 & -4.8 \\
Glucose & 56.9 & $-0.62 \pm 5.83$ & $-3.27 \pm 7.59$ & & \\
\hline
\end{tabular}

*Significant $\mathrm{P}$ values $<0.05$.

$\mathrm{OP}=$ overweight placebo, $\mathrm{OT}=$ overweight treatment, $\mathrm{NP}=$ normal weight placebo and $\mathrm{NT}=$ normal weight treatment.

Values at the start of the study (Time 0 ) listed in the first column are means of data from individuals. Mean changes after 4 weeks and 8 weeks are listed in the second and third columns as \pm Standard Deviation. A negative value indicated a decrease in value and a positive number represents an increase in value. $\% \Delta$ values are percent mean change in value between time 0 and either 4 or 8 weeks. Values* are significant $P$-value comparisons $(<0.05)$ between initial values at Time 0 and either 4 weeks or 8 weeks. The unit of measurement for blood pressure is $\mathrm{mmHg}$ and for glucose is $\mathrm{mg} / \mathrm{dl}$. 


\section{Fasting Glucose}

In Group OT, fasting glucose levels decreased significantly at 4 weeks $(9.5 \%)$ and 8 weeks $(19.6 \%)$ compared to baseline ( $\mathrm{p}<0.05$ and $\mathrm{p}<0.001$, respectively) (Table 6 ). There were no significant changes in fasting glucose levels in Group OP. Comparison of overweight groups showed a significant difference between treatment and placebo at both 4 and 8 weeks (both $\mathrm{p}<0.001$ ) (Table 4).

In Groups NT and NP, fasting glucose levels did not change significantly from the beginning of the study and there were no significant differences between the two normal weight groups.

\section{Discussion}

NP 06-1 was administered to the trial participants with a dose of 4 capsules $(1,480 \mathrm{mg})$ per day with no significant adverse events reported; an indication of safety.

NP06-1 contains a Phellodendron amurense tree bark extract standardized to a consistent amount of berberine. NP061 also contains a Citrus sinensis (orange) peel which contains bioflavonoids, including polymethoxylated flavones (PMFs). Both berberine and PMFs have demonstrated beneficial effects on lipid levels as well as glucose tolerance.

Berberine has been shown in human clinical studies to have beneficial effects on lipid levels: reducing triglycerides and LDL-cholesterol and in one study increasing HDL-cholesterol [6,7]. Berberine may affect lipid levels by increasing expression of hepatic low density lipoprotein receptor [8]. In a hamster model, citrus PMFs caused significant reductions in total cholesterol ranging from 19 to 27\%; and in very low density cholesterol (VLDL-C) and low density cholesterol (LDL-C), ranging from 32 to $40 \%$ [9].

NP 06-1 generally improved lipid levels in this study, which is consistent with literature reports for berberine and PMFs. In this study, the overweight and normal weight treatment groups had significant reductions in triglycerides and LDL-cholesterol compared to their matched control groups. For HDL-cholesterol, there was a significant increase compared to placebo for both treatment groups. The greatest change in lipid levels was observed for LDL in the overweight treatment group, which decreased by an impressive $44.6 \%$. These results indicate that NP06-1 may have a beneficial effect on cardiovascular risk factors. High triglycerides, total cholesterol and LDL-cholesterol levels, along with low HDL-cholesterol levels, have been established as indicators of risk for cardiovascular disease [10].
Another risk factor for cardiovascular disease is high blood pressure [11]. NP06-1 caused significant decreases in blood pressure in both overweight and normal weight treatment groups compared to the start of the study and compared to their respective placebos. There was also a significant decrease in fasting glucose levels in the overweight treatment group compared to the start of the study and to the overweight placebo group. There was no change in fasting blood glucose for either normal weight group.

Both overweight and normal weight treatment groups lost a significant amount of weight compared to the start of the study and to their respective placebo groups. The overweight treatment group lost an average of $5.1 \%$ body weight after 8 weeks. Weight loss has previously been reported to decrease fasting glucose levels [12]. Thus weight loss may have influenced the decrease in fasting glucose levels observed in this study. The weight loss appears to be due to NP06-1 as participants were instructed not to change their exercise or diet regimens. Further, berberine has been shown to reduce body weight and to improve glucose tolerance. A mechanism for this is suggested to be an increase in AMP-activated protein kinase activity in adipocytes [13].

PMFs have also been shown to decrease insulin levels and improve glucose tolerance in hamsters [14]. Research is ongoing on the potential antiatherogenic effects of citrus flavonoids $[15,16]$.

\section{Conclusion}

In a placebo-controlled pilot clinical study, NP06-1 offered several potential health benefits in normal and overweight subjects with osteoarthritis of the knee. These potential benefits include significant improvements in cardiovascular risk factors; namely lipid levels and blood pressure. There appears to have been additional benefits to the overweight group compared to the normal weight group in decreases in fasting glucose. Treatment-induced weight loss was also observed.

\section{Competing interests}

This study was sponsored by Next Pharmaceuticals. Mr Garrison was President and CEO. Dr. Chambliss and Mr. Kothari were compensated as consultants. Miss Dolnick is employed by Next Pharmaceuticals.

\section{Authors' contributions}

All authors were involved in the design of the study, as well as analysis and interpretation of the data. The study was carried out by Dr. JO, the Principal Investigator and Miss EE, his assistant. 


\section{Acknowledgements}

We thank Marilyn Barrett PhD, who provided medical writing on behalf of Next Pharmaceuticals.

\section{References}

I. Bijlsma JW, Knahr K: Strategies for the prevention and management of osteoarthritis of the hip and knee. Best Pract Res Clin Rheumatol 2007, 2 I(I):59-76.

2. Ameye LG, Chee WS: Osteoarthritis and nutrition. From nutraceuticals to functional foods: a systematic review of the scientific evidence. Arthritis Res Ther 2006, 8(4):RI 27.

3. Long L, Soeken K, Ernst E: Herbal medicines for the treatment of osteoarthritis: a systematic review. Rheumatology 200I, 40:779-793.

4. Christensen R, Bartels EM, Astrup A, Bliddal H: Effect of weight reduction in obese patients diagnosed with knee osteoarthritis: a systematic review and meta-analysis. Ann Rheum Dis 2007, 66(4):433-9.

5. Appel SJ, Jones ED, Kennedy-Malone L: Central obesity and the metabolic syndrome: implications for primary care providers. J Am Acad Nurse Pract 2004, I 6(8):335-342.

6. Kong W, Wei J, Abidi P, Lin M, Inaba S, Li C, Wang Y, Wang Z, Si S, Pan H, Wang S, Wu J, Wang $Y$, Li Z, Liu J, Jiang JD: Berberine is a novel cholesterol-lowering drug working through a unique mechanism distinct from statins. Nat Med 2004, I 0( I 2):|344-5|.

7. Cicero AF, Rovati LC, Setnikar I: Eulipidemic effects of berberine administered alone or in combination with other natural cholesterol-lowering agents. A single-blind clinical investigation. Arzneimittelforschung 2007, 57(I):26-30.

8. Brusq JM, Ancellin N, Grondin P, Guillard R, Martin S, Saintillan Y, Issandou $M$ : Inhibition of lipid synthesis through activation of AMP kinase: an additional mechanism for the hypolipidemic effects of berberine. J Lipid Res 2006, 47(6): I28I-8.

9. Kurowska EM, Manthey JA: Hypolipidemic effects and absorption of citrus polymethoxylated flavones in hamsters with diet-induced hypercholesterolemia. I Agric Food Chem 2004, 52(I 0):2879-86.

10. Stone NJ, Bilek S, Rosenbaum S: Recent National Cholesterol Education Program Adult Treatment Panel III update: adjustments and options. Am J Cardiol 2005, 96(4A):53E-59E.

II. Jamerson KA, Basile J: Prompt, aggressive BP lowering in highrisk patients. J Clin Hypertens (Greenwich) 2008, IO(I Suppl I):40-8.

12. Gagliardi L, Wittert G: Management of obesity in patients with type 2 diabetes mellitus. Curr Diabetes Rev 2007, 3(2):95-I0.

13. Lee YS, Kim WS, Kim KH, Yoon MJ, Cho HJ, Shen Y, Ye JM, Lee CH, Oh WK, Kim CT, Hohnen-Behrens C, Gosby A, Kraegen EW, James $\mathrm{DE}, \mathrm{Kim}$ JB: Berberine, a natural plant product, activates AMP-activated protein kinase with beneficial metabolic effects in diabetic and insulin-resistant states. Diabetes 2006 , 55(8):2256-64.

14. Li, Li RW, Theriault AG, Au K, Douglas TD, Casaschi A, Kurowska $E M$, Mukherjee $R$, et al:: Citrus polymethoxylated flavones improve lipid and glucose homeostasis and modulate adipocytokines in fructose-induced insulin resistant hamsters. Life Sci 2006, 79(4):365-73.

15. Kurowska EM, Manthey JA, Casaschi A, Theriault AG: Modulation of Hepg 2 cell net apolipoprotein B secretion by the Citrus polymethoxyflavone, tangeretin. Lipids 2004, 39(2): |43-I5I.

16. Whitman SC, Kurowska EM, Manthey JA, Daugherty A: Nobiletin, a citrus flavonoid isolated from tangerines, selectively inhibits class A scavenger receptor-mediated metabolism of acetylated LDL by mouse macrophages. Atherosclerosis 2005, I 78(I):25-32.

\section{Publish with Bio Med Central and every scientist can read your work free of charge}

"BioMed Central will be the most significant development for disseminating the results of biomedical research in our lifetime. "

Sir Paul Nurse, Cancer Research UK

Your research papers will be:

- available free of charge to the entire biomedical community

- peer reviewed and published immediately upon acceptance

- cited in PubMed and archived on PubMed Central

- yours - you keep the copyright

Submit your manuscript here:

http://www.biomedcentral.com/info/publishing_adv.asp
BiolMedcentral 